\title{
Reconstrucción de la vía biliar, secundaria a lesiones mayores iatrogénicas
}

\author{
Reconstruction of the biliary tract, \\ secondary to iatrogenic major injuries
}

Jorge Elorza Bonifaz Calvo, ${ }^{*}$ Amado Fernando Palacio Vélez*

Palabras clave:

Colédoco, lesión, reconstrucción, vía biliar.

Key words: Bile duct, common bile duct, injury, reconstruction.

\section{RESUMEN}

Antecedentes: La lesión de vía biliar es una de las complicaciones más temidas, peligrosas y que aumentan la morbimortalidad. Con la colecistectomía laparoscópica se incrementó la incidencia de lesión hasta que se estabilizó en un $1.3 \%$, lo cual es aún alto para el $0.6 \%$ de la era abierta. El objetivo es mostrar la experiencia de la unidad hospitalaria en el manejo de la lesión de vía biliar en un periodo de 30 años. Material y métodos: Éste es un estudio retrospectivo, descriptivo, observacional en pacientes del Servicio de Cirugía General del Hospital Regional "General Ignacio Zaragoza" en el periodo comprendido de 1980 a 2009, con análisis de expedientes clínicos, estudios de laboratorio e imágenes de control y seguimiento de los pacientes intervenidos quirúrgicamente. Se incluyeron, solamente, individuos con lesiones mayores de vía biliar con diagnóstico trans- y postoperatorio sometidos a reconstrucción de la misma. Se intervinieron 67 sujetos, de los cuales 58 eran mujeres y nueve hombres. Resultados: El tipo de lesión más frecuente fue Bismuth III y el procedimiento quirúrgico más utilizado fue la hepaticoyeyunoanastomosis en Y de Roux, en 54 de ellos, con un $5.2 \%$ de mortalidad en los primeros 13 meses y un $89.5 \%$ de éxito en el primer año de seguimiento. Conclusiones: La incidencia de la lesión de la vía biliar durante un procedimiento quirúrgico es baja en centros de concentración y alta especialidad. Es imperativo para el cirujano conocer las alternativas diagnósticas y quirúrgicas al enfrentar este tipo de lesiones.

\section{ABSTRACT}

Background: Bile duct injury is one of the most feared and dangerous complications, which leads to high morbidity and mortality. The incidence of common bile duct injuries during laparoscopic cholecystectomy, increased until it reached $1.3 \%$, which is still higher than the $0.6 \%$ from the open surgery era. The aim of this study is to show our experience in reconstruction of bile duct injuries over a 30-year period. Material and methods: This is a retrospective, descriptive, and observational study on patients from the General Surgery department of the Regional Hospital "General Ignacio Zaragoza", during the period between 1980 and 2009, in which the patients' clinical histories, laboratory and radiology studies, and follow-up analysis were performed. Only the patients with a major bile duct injury diagnosed during or after the surgery were included. Sixty-seven patients were included, 58 women, and nine men. Results: The most common type of injury was Bismuth III, and the surgical procedure was Roux-en-Y hepaticojejunostomy in 54 patients, with $5.2 \%$ of mortality within the first 13 months, and $89.5 \%$ success during the first year of follow-up. Conclusion: Common bile duct injury during a surgical procedure is low in highly specialized hospitals. It is necessary to know the diagnosis and surgical options due to the elevated morbidity and mortality rates. There must be a close survival follow-up for complications, as cholangitis may appear.
* Servicio de Cirugía General, Hospital Regional "General Ignacio Zaragoza", Instituto de Seguridad y Servicios Sociales de los Trabajadores del Estado (ISSSTE). México, DF.

Recibido: 01/01/2015 Aceptado: 01/05/2015

\section{ANTECEDENTES}

$\mathrm{L}^{\mathrm{a}}$ a lesión de vía biliar es una de las complicaciones más temidas, peligrosas y conlleva un aumento en la morbimortalidad del enfermo. Se define como la obstrucción, sección parcial o total de la vía biliar principal o de los conductos aberrantes que drenan a la misma o algún sector hepático.
Desde el origen de la colecistectomía laparoscópica, se incrementó la incidencia de lesión de vía biliar hasta que se estabilizó en un $1.3 \%$, lo cual es aún alto para el $0.6 \%$ de la era abierta.

Bachelier y colaboradores ${ }^{1}$ presentan como referencia la incidencia de lesión de vía biliar en colecistectomía laparoscópica de $0.6 \%$, la cual es de casi el doble que en el procedimiento 
abierto, cifra que se confirma en centros de todos los niveles, como en Grecia, por Zacharakis y su grupo ${ }^{2}$ en Tesalónica en su revisión de 1,042 pacientes entre 1992 y 2004, aunque no reportaron mortalidad alguna.

Algunos autores como Fletcher, Calvete y MacFadyen en la revisión de De Reuver y colegas ${ }^{3}$ hablan de una incidencia de entre 0.3 y $1.4 \%$.

Moossa y su equipo, ${ }^{4}$ en 1992, publican una serie que refiere hasta un $7 \%$ de incidencia por la vía laparoscópica.

Bergman y colaboradores ${ }^{5}$ publicaron en 1996 una serie de 53 pacientes, en cinco años, sometidos a colecistectomía laparoscópica con lesión de vía biliar; 48 fueron referidos al Centro Médico Académico de Ámsterdam, donde se estableció una de las primeras clasificaciones.

El diagnóstico se lleva a cabo en el transoperatorio en un $15-50 \%$ de los casos. ${ }^{6-10}$

Stabile ${ }^{11}$ mencionó en 1998 tres factores de suma importancia que contribuyen en la etiología de la lesión de la vía biliar:

1. Anatomía

2. Patología

3. Cirugía

Esto confirma lo predicho por Johnston en 1986, quien mencionó que una anatomía peligrosa, patologías peligrosas y una cirugía peligrosa son factores que llevan a lesiones de vía biliar tanto en colecistectomía abierta como en laparoscópica. ${ }^{12}$ Todas y cada una de ellas tienen un alto riesgo en sus distintas variantes.

Han sido publicadas innumerables series mostrando la incidencia, las distintas alternativas de tratamiento quirúrgico y la morbimortalidad. Uno de los primeros estudios prospectivos fue el de Smith, ${ }^{13}$ con seguimiento de 15 años a 4,425 pacientes sometidos a colecistectomía, refiriendo hasta un $6.3 \%$ de complicaciones.

Múltiples centros han reportado series de casos con lesiones de vía biliar, pero pocos han sido aquellos que incluyen, de manera simultánea, el impacto de las lesiones vasculares. La reciente importancia de la isquemia en la vía biliar tras una anastomosis bilioentérica ha sido tema fundamental hasta en procedimientos como trasplante hepático. En dicho caso, esto conlleva a una elevación de la morbimortalidad.

Existen clasificaciones como la de Bismuth y la de Strasberg que no contemplan la concomitancia de la lesión de vía biliar y su irrigación. Otro ejemplo es la clasificación de la severidad de la lesión propuesta por Keulemans y su grupo $^{14}$ en 1998, dando a conocer los criterios de Ámsterdam, que tampoco contempla la lesión arterial, únicamente aquella de vía biliar.

Desde 2001 se realizaron los primeros estudios que señalan la importancia y simultaneidad de la lesión de vía biliar y su irrigación, junto con la falla en la reconstrucción anastomótica ulterior.

Buell y colegas ${ }^{15}$ volvieron a mencionar en 2002 esta relación con el nivel de lesión de la vía biliar (Bismuth), haciendo referencia a una mayor incidencia en los niveles altos de lesión según dicha clasificación. En esta serie, se mencionó tanto la estenosis arterial hepática como la sección completa de la misma. Recomiendan, para su identificación, realizar angiografía preoperatoria.

En casos de lesión aislada de la arteria hepática derecha, el lóbulo derecho llega a recibir irrigación colateral a partir del lóbulo izquierdo hasta en un $30 \%$ de los casos, presentándose necrosis en aquellos que no la reciben, lo cual resulta del aprendizaje en trasplante hepático al momento de colocar injertos totales con falla, resultado de una trombosis de la arteria hepática en una etapa temprana. Esto está presente en aquellos intervenidos en varias ocasiones, que presentaron lesión arterial desde el primer procedimiento o en los subsecuentes.

Way y su equipo ${ }^{16}$ proponen una clasificación laparoscópica de las lesiones de la vía biliar (Cuadro I).

Ésta es la primera clasificación en donde se contempla la sección de los conductos biliares principales y la relación con la lesión en su irrigación, en este caso, con la arteria hepática derecha, contemplando las variantes anatómicas que pueda presentar.

Asimismo, la clasificación de Hannover, propuesta por Bektas y colaboradores ${ }^{17}$ en 2007, trata de unificar criterios; en ella se menciona lo ya sabido, como que la clasificación de Strasberg y la de Neuhaus no contemplan el 


\section{Cuadro I. Clasificación propuesta por Way y colaboradores. ${ }^{16}$}

\section{Table 1. Mechanism of injury}

Class I CBD mistaken for cystic duct, but recognized Cholangiogram incision in cystic duct extended into CBD

Class II Lateral damage to the CHD from cautery or clips placed on duct Associated bleeding, poor visibility

Class III CBD mistaken for cystic duct, not recognized CBD, CHD, R, L hepatic ducts transected and/or resected

Class IV RHD mistaken for cystic duct, RHA mistaken for cystic artery, RHD and RHA transected Lateral damage to the RHD from cautery or clips placed on duct

compromiso vascular, y la de Stewart y Way y los sistemas de Siewert y Neuhaus no distinguen entre las lesiones a nivel y las que se encuentran por arriba de la confluencia de los conductos hepáticos. ${ }^{18,19}$

Según Alves y su grupo, ${ }^{20}$ se ha estimado un $7 \%$ de lesión a la arteria hepática en cadáveres, encontrándose esta relación aumentada en aquellos pacientes con lesión de vía biliar con un rango del 12 al 39\%.

De Santibáñes y colegas $^{21}$ hacen hincapié en el uso de angiografía abdominal en los casos de sospecha de lesión arterial, con especial atención en la posible lesión a la arteria hepática y al árbol portal.

Caso de especial importancia, tal y como lo describen Babel y su equipo, ${ }^{22}$ es la variabilidad anatómica, como en el caso de los conductos hepáticos sectoriales derechos, las cuales se llegan a presentar en un 15-20\% de los pacientes. ${ }^{23}$ Mencionan tres casos en los que se lesiona el conducto hepático derecho posterior del tipo Strasberg C.

Fragulidis y colaboradores ${ }^{24}$ hacen mención de la frecuencia con que se lesiona la vía biliar por su variabilidad anatómica, especialmente en la confluencia de los conductos hepáticos, la más frecuente es hacia el conducto hepático derecho posterior. En su muestra de 234 pacientes, refiere a siete de ellos. Incluso, describe una clasificación propuesta por Ayuso y su grupo ${ }^{25}$ para dichas variantes, apoyado en estudios de imagenología, como la resonancia magnética colangiopancreática.

Colovic ${ }^{23}$ no es la excepción y muestra una serie de 19 casos con variantes entre el conducto cístico y los hepáticos sectoriales, específicamente el V.

Dentro de las tres variantes más frecuentes se encuentran las siguientes:

1. La desembocadura del cístico cerca del sectorial hacia el hepático común.

2. La desembocadura hacia el conducto hepático sectorial (no hacia el hepático común).

3. La convergencia entre la desembocadura del conducto hepático sectorial y el cístico.

Desde 1995, Strasberg y colegas $^{26}$ mencionaron la necesidad de tener una "visión crítica de seguridad".

\section{La percepción visual provee una estimación de la realidad, no una réplica. ${ }^{16}$}

Las expectativas hacia un entrenamiento efectivo recaen en que el desempeño humano no puede llevarse a un punto de perfección; el error o falla esporádica son inevitables $y$, frecuentemente, predecibles. Aun más importante, las heurísticas y los prejuicios no pueden ser borrados o extraídos de la decisión humana.

Archer y su equipo ${ }^{27}$ hacen referencia a la experiencia de los médicos cuyo entrenamiento laparoscópico fue después de la residencia y aquéllos en los que fue durante la misma; concluyen que los primeros tuvieron mayor incidencia de lesión a la vía biliar, pero que completando la curva de aprendizaje de un número de 200, la incidencia se equiparó en ambos grupos. 
Tay, ${ }^{28}$ en Singapur, menciona la importancia de un plan de estudios y un entrenamiento quirúrgico adecuados, con supervisión estrecha por un cirujano, que reditúa en resultados óptimos y menor riesgo para el paciente. Éste es uno de los primeros estudios en donde se menciona el cuidado al realizar la disección con energía, por el efecto colateral que conlleva al propagarse el incremento de temperatura local, el cual es corroborado por Smith. ${ }^{29}$ También, consideran como estándar de oro la colecistectomía con colangiografía intraoperatoria, además de ser una medida de seguridad. Ésta es el recurso tecnológico más valioso y práctico para verificar alteraciones o variantes anatómicas en esta región.

De Reuver y colaboradores, ${ }^{30}$ en 2007, hacen hincapié en los excelentes resultados en individuos referidos a una unidad de tercer nivel con experiencia quirúrgica y recursos necesarios para el manejo del paciente; así como en la nula mortalidad en caso de que el paciente fuera referido y la reconstrucción de la vía biliar se hubiera llevado a cabo de manera tardía, con menor incidencia de complicaciones, hasta en seis semanas después del procedimiento inicial.

En la opinión de De Santibáñes y su grupo, ${ }^{21}$ la espera e intervención quirúrgica tardía de hasta seis u ocho semanas desde el procedimiento inicial, en aquellos casos con lesión térmica, sólo agrava el tipo de lesión, haciendo más difícil la reconstrucción de la vía biliar.

El Dr. Lillemoe hace énfasis en la importancia de contar con un servicio de radiología intervencionista eficiente y con experiencia, que funciona como antesala o terapéutica definitiva. ${ }^{31}$

El manejo de la lesión de vía biliar es un tema controvertido actualmente, con referencia al tipo de reconstrucción. El principal objetivo de ésta, ya sea de manera primaria o secundaria (en casos de múltiples intentos de reparación), es la reconstrucción del tracto biliar o la anastomosis de mucosa biliar hacia la mucosa intestinal. El manejo de la lesión de vía biliar también va encaminado a evitar complicaciones mediante el drenaje de biliperitoneo, ya sea percutáneo o por vía laparoscópica. Existen autores que consideran necesaria la reparación posterior a un mes para disminuir la inflamación en los tejidos y permitir una mejor disección de los mismos, así como el drenaje de la vía biliar. Un segundo procedimiento quirúrgico de reparación de la vía biliar se asocia con más complicaciones del tipo estenosis $\mathrm{y} / \mathrm{u}$ oclusiones en la misma.

Bismuth y sus colegas llegaron a notar que el nivel de estenosis durante la reparación era más alto que el original durante la lesión. ${ }^{32}$ Durante un segundo tiempo quirúrgico, por mala reparación de la vía biliar, el nivel de estenosis era todavía mucho más alto. ${ }^{33}$

De Santibáñes y su equipo ${ }^{21}$ mencionan la estenosis alta en aquellos pacientes ya sometidos a un primer procedimiento de reconstrucción o reparación de la vía biliar como de alta complejidad, con morbimortalidad alta por la tendencia de los tejidos hacia la fibrosis, resultando en la mayoría de los pacientes con hipertensión portal.

La reconstrucción terminoterminal es difícil de llevar a cabo, incluso al no existir gran pérdida de tejido, y raramente posible por la tensión y el tamaño de los muñones o extremos de la vía biliar. La reconstrucción que se prefiere es la hepaticoyeyunoanastomosis en $\mathrm{Y}$ de Roux. El asa debe ser de alrededor de $70 \mathrm{~cm}$ para así evitar el reflujo alimentario hacia el árbol biliar a través del mesocolon mediante una ventana avascular hacia la derecha del duodeno. Las adherencias entre el lóbulo cuadrado y el pedículo hepático suelen dar la apariencia de una retracción del muñón proximal del árbol biliar. La disección adecuada de este lóbulo hepático entre el colon y el duodeno suele restablecer una longitud adecuada del pedículo hepático. Incluso, la exteriorización de este lóbulo de su aparente posición y localización intrahepática se logra a través de la disección y separación del lóbulo desde el pedículo hepático. Lo que dicta una reconstrucción exitosa es la longitud de la mucosa biliar sana en la anastomosis con el yeyuno.

En el algoritmo, De Santibáñes ${ }^{21}$ (Figura 1) hace referencia al tipo de reconstrucción durante el transoperatorio. En caso de no realizarse en el mismo tiempo quirúrgico, se recomienda la colocación de drenajes subfrénico e hiliares para su pronta canalización y traslado a un centro de especialidades, evitando ligar conductos biliares por el riesgo de colangitis, así como de 


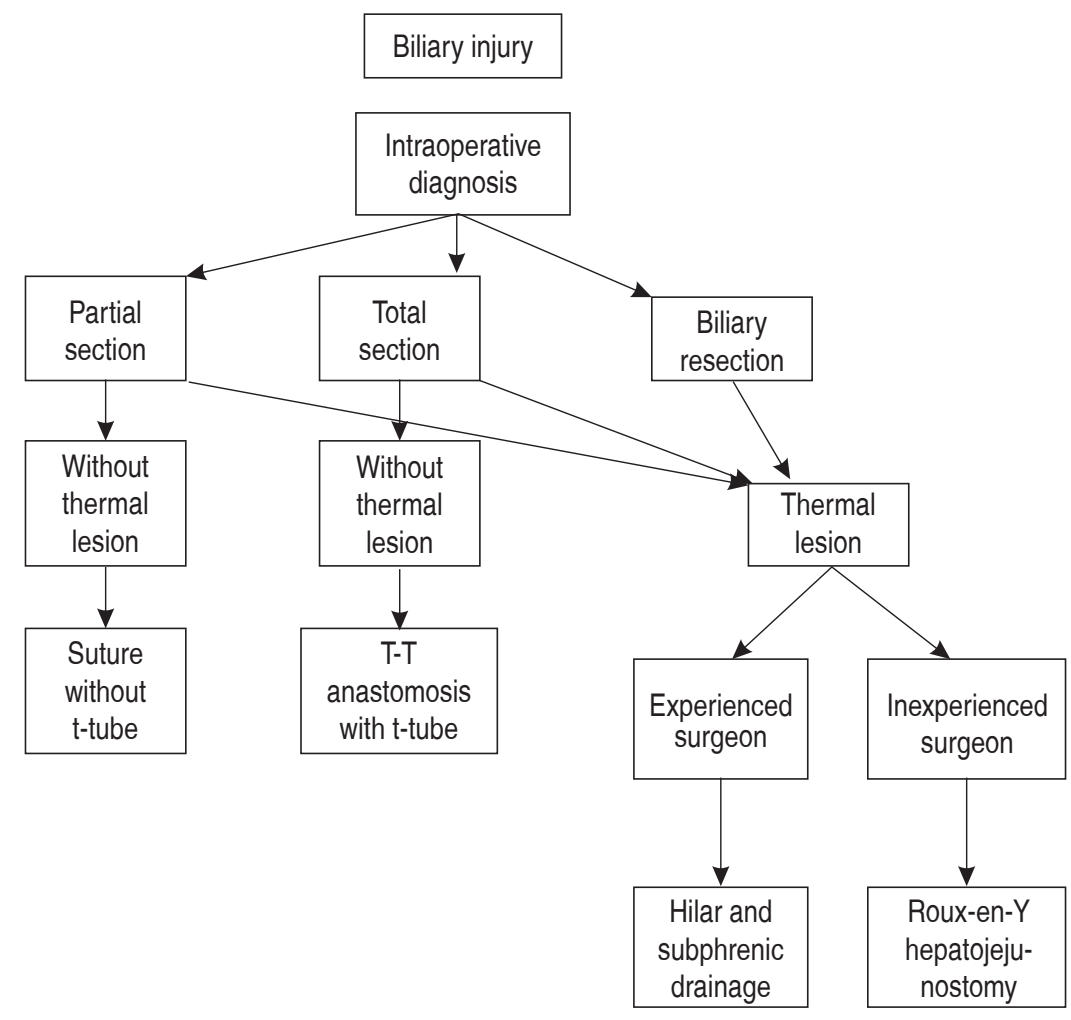

Figura 1.

Manejo para lesiones de vía biliar diagnosticadas durante transoperatorio. ${ }^{52}$

fuga biliar al momento de retirar la ligadura en una cirugía ulterior.

En lesiones complejas como las de los pacientes ya sometidos a un procedimiento quirúrgico previo, explica la posibilidad de resecciones hepáticas, en conjunto con una terapéutica radiológica-intervencionista.

Jablonska y Lampe ${ }^{34}$ mencionan en una extensa revisión posibilidades como la hepaticoyeyunoanastomosis, la anastomosis terminoterminal de la vía biliar en caso de que ésta no sea menor a $4 \mathrm{~mm}$ de diámetro (con material monofilamento 5-0, aguja atraumática), y la coledocoduodenostomía cuando el colédoco posee un diámetro ancho de hasta $15 \mathrm{~mm}$. En este último caso, Tocchi y colaboradores ${ }^{35}$ analizan la incidencia de malignidad en los casos de coledocoduodenostomía versus hepaticoyeyunoanastomosis en un periodo de 30 años, con $7.6 \%$ versus $1.9 \%$, respectivamente.

Fischer y su grupo, ${ }^{36}$ en una serie de 44 pacientes, concluyen que mientras más tiempo transcurra entre el procedimiento inicial y la intervención definitiva habrá más probabilidad de complicaciones y estancia hospitalaria en la unidad de cuidados intensivos, que en aquellos referidos en las primeras 72 horas.

El uso y duración de los drenajes biliares es controversial, aunque el propósito es limitar el proceso inflamatorio y la fibrosis posteriores al procedimiento quirúrgico.

Jablonska y Lampe, ${ }^{34}$ haciendo referencia a estudios previos de Lillemoe y sus colegas, ${ }^{37,38}$ mencionan que la permanencia de drenajes biliares por más de tres meses no ha demostrado beneficio alguno.

Mercado y su equipo ${ }^{39}$ recomiendan la colocación de stents transanastomóticos cuando se cuenta con una vía biliar no mayor a 4 $\mathrm{mm}$ de diámetro y cuando se observa tejido inflamatorio en los extremos de los bordes anastomóticos, lo cual pone en riesgo y en duda el éxito de la anastomosis.

De acuerdo con algunos autores, la morbilidad ronda el $20-30 \%$ y la mortalidad, el $0-2 \% .31,40,41$

La infección de la herida quirúrgica es la complicación más frecuente (8-17.7\%). Otras son biloma, abscesos, dehiscencia de las anastomosis, 
fístula biliar, colangitis, peritonitis, eventración, neumonía, hemorragia de tubo digestivo, sepsis, pancreatitis, complicaciones trombóticas y embólicas, íleo, falla orgánica múltiple.

Sólo en aquellos pacientes referidos con colangitis y fístula biliar se emplea el drenaje percutáneo transhepático. Para ello, se debe contar con un servicio de radiología intervencionista y endoscopia con experiencia en los procedimientos requeridos.

Dentro de las secuelas corroboradas por biopsia hepática se encuentra la fibrosis hepática, la cual, según Johnson y colaboradores, ${ }^{42}$ se debe al retraso en el tratamiento resolutivo para el paciente.

Entre otras, se menciona la aparición de cirrosis biliar secundaria a consecuencia de estenosis biliar primaria en un periodo promedio de 7.1 años; 4.6 años con el antecedente de litiasis y 0.8 años con el de malignidad.

La prueba de éxito del tratamiento quirúrgico es la ausencia de estenosis en el sitio de la anastomosis. En algunos centros de referencia, la tasa de éxito se encuentra en el 70-90\%. Dos terceras partes (65\%) de las estenosis biliares recurrentes se llegan a desarrollar en los primeros 2-3 años posteriores a la reconstrucción, $80 \%$ a los 5 y $90 \%$ a los 7 años. Existen algunas estenosis referidas en la literatura hasta 10 años después. Por lo anterior, es necesario un seguimiento estrecho a largo plazo. ${ }^{34,37,38,40,43,44}$

Pocas son las referencias bibliográficas referentes al estilo y calidad de vida de los pacientes con antecedente de lesión de vía biliar y su reparación. Boerma y su grupo ${ }^{45}$ hacen un seguimiento a 106 individuos durante 70 meses en cuanto a su calidad de vida desde el momento de la detección de la lesión. Junto con De Reuver y sus colegas, en $2007,{ }^{46}$ describen una serie de encuestas en cuanto a la calidad de vida y secuelas de los pacientes, como función física, dolor, vitalidad, función mental, salud mental, salud en general; observan discreta disminución del rendimiento en cada una de estas variables para los sujetos con lesión de la vía biliar y una terapéutica resolutiva.

Terblanche y su equipo ${ }^{44}$ ofrecen una de las clasificaciones con mayor utilidad en cuanto a la evaluación del éxito a largo plazo basada en sintomatología biliar clínica. Nielubowicz, Lygidakis, Muñoz y McDonald son algunas otras clasificaciones descritas en la literatura. Anders- son y colaboradores ${ }^{47}$ incluso hacen una última referencia del impacto de calidad de vida a largo plazo, el cual no se ha estudiado a fondo.

El costo promedio de cada paciente va desde los 21,837 hasta los 107,568 euros.

Un aspecto importante es el costo económico, ya referido desde 1992 por Smith, ${ }^{29}$ el cual es revisado y ampliado por autores como Savader y colaboradores, en $1997 .{ }^{48}$ En dicha revisión, los costos fueron desde $\$ 2,800$ hasta $\$ 305,000$ dólares americanos por internamiento de cada enfermo.

\section{MATERIAL Y MÉTODOS}

Se trata de un estudio retrospectivo, descriptivo y observacional. Se llevó a cabo en pacientes del Servicio de Cirugía General del Hospital Regional "General Ignacio Zaragoza" del Instituto de Seguridad y Servicios Sociales de los Trabajadores del Estado (ISSSTE), en un periodo comprendido de 1980 a 2009, con análisis de los expedientes clínicos, estudios de laboratorio e imagen de control y seguimiento de los individuos intervenidos quirúrgicamente.

Se incluyeron todos los sujetos intervenidos quirúrgicamente de colecistectomía abierta y/o laparoscópica con lesiones mayores de vía biliar, con diagnóstico trans- y postoperatorio y reconstrucción de la misma en el periodo comprendido entre 1980 y 2009. Las variables y las unidades de medida a considerar fueron edad, enfermos referidos, pacientes operados en el hospital, tipos de lesión de la vía biliar, procedimientos quirúrgicos de reconstrucción

Cuadro II. Distribución de edades.

\begin{tabular}{ccr} 
Edad (años) & $\begin{array}{c}\text { Pacientes } \\
\text { (número) }\end{array}$ & \multicolumn{1}{c}{$\%$} \\
\hline $10-19$ & 1 & 1.5 \\
$20-29$ & 17 & 25.4 \\
$30-39$ & 21 & 31.3 \\
$40-49$ & 15 & 22.4 \\
$50-59$ & 9 & 13.4 \\
$60-69$ & 3 & 4.5 \\
$70-79$ & 1 & 1.5 \\
Media: 39 & 67 & 100.0
\end{tabular}




\begin{tabular}{lcr}
\multicolumn{3}{c}{ Cuadro III. Métodos diagnósticos. } \\
\hline Métodos diagnósticos & $\begin{array}{c}\text { Procedimientos } \\
\text { diagnósticos }\end{array}$ & $\mathbf{\%}$ \\
\hline Colangiografía percutánea & 32 & 45.7 \\
Colangiografía retrógrada endoscópica & 16 & 22.8 \\
Resonancia magnética hepatobiliar & 6 & 8.6 \\
Colangiografía dinámica con HIDA(99 Tc) & 2 & 2.9 \\
Diagnóstico transoperatorio & 14 & 20.0 \\
& 70 & 100.0
\end{tabular}

\section{Cuadro IV. Tipos de lesión (clasificación de Bismuth).}

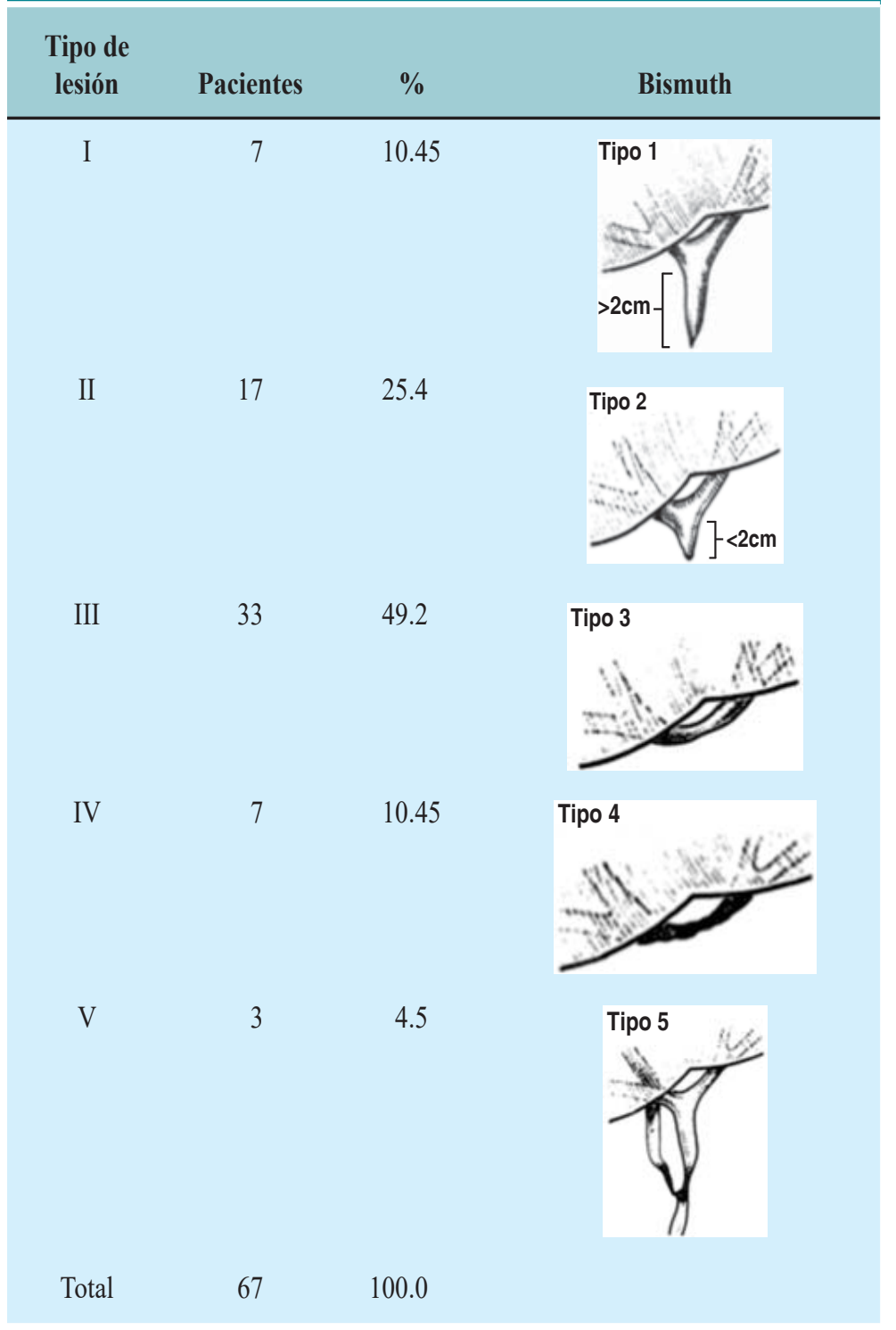

de la vía biliar realizados, tipos de complicación en el postoperatorio y mortalidad.

\section{RESULTADOS}

En un periodo de 30 años (1980-2009), se intervinieron 67 personas, de las cuales 58 eran mujeres y nueve hombres, en una relación aproximada de 6:1.

El rango de edad era variable y se muestra en el cuadro II.

Dentro del tipo de médicos que causaron la lesión, se encontraron 58 cirujanos y residentes de cirugía general, siete médicos generales y dos ginecólogos.

Dicha lesión ocurrió en 46 procedimientos abiertos y 21 laparoscópicos.

Del total de pacientes, 43 fueron referidos por otras unidades hospitalarias, y 24 fueron de la propia institución ( $0.4 \%$ en 11,415 procedimientos).

Solamente en catorce individuos (20.9\%) se identificó la iatrogenia durante el procedimiento quirúrgico, mientras que en 53 (79.1\%) se detectó en un lapso de hasta 30 días posteriores a la cirugía inicial.

Los métodos de diagnóstico utilizados se exponen en el cuadro III.

El tipo de lesión (mediante la clasificación de Bismuth) se describe en el cuadro IV.

El tipo de cirugía realizada se describe en el cuadro V. Cabe señalar que se realizó biopsia hepática a la mayoría de los sujetos; por ello, en el total no se incluye dicho procedimiento como uno separado, sino como el mismo en cada enfermo.

Las complicaciones presentes en los pacientes se presentan en el cuadro VI.

Dentro de las comorbilidades en nuestra muestra, se encontraron las reflejadas en el cuadro VII.

El seguimiento a largo plazo arrojó los siguientes resultados:

I. Ocho con mortalidad no relacionada con el procedimiento quirúrgico (11.9\%).

II. Dos con colangitis posterior al tercer año de la cirugía (3\%).

III. Cincuenta y tres vivos sin complicaciones (79.1\%). 


\section{Cuadro V. Tipos de cirugía realizadas.}

\begin{tabular}{lcc}
\hline Operación realizada & Número & \% \\
\hline Biopsia hepática & 66 & 94.3 \\
Intrahepaticoyeyunoanastomosis alta (Y de Roux) & 29 & 41.4 \\
Hepaticoyeyunoanastomosis baja (Y de Roux) & 26 & 37.1 \\
Conversión de anastomosis baja a alta (Y de Roux, reop. por estenosis) & 3 & 4.3 \\
Anastomosis terminoterminal & 4 & 5.7 \\
Procedimiento de Longmire & 1 & 1.4 \\
Portoenterostomía (Y de Roux) & 6 & 8.6 \\
Exploración y biopsia & 1 & 1.4 \\
Total & 70 & 100.0
\end{tabular}

\begin{tabular}{|lcc}
\hline \multicolumn{3}{c}{ Cuadro VI. Complicaciones. } \\
\hline Complicaciones & Pacientes & $\%$ \\
\hline Infección de herida & 2 & 2.9 \\
Colangitis & 3 & 4.3 \\
Absceso subfrénico & 2 & 2.9 \\
Eventración & 2 & 2.9 \\
Ruptura de sonda T & 1 & 1.4 \\
Fístula biliar & 1 & 1.4 \\
Neumonía & 2 & 2.9 \\
& 13 & 18.7
\end{tabular}

En cuanto a la mortalidad relacionada con el procedimiento quirúrgico, los resultados fueron de un paciente $(1.4 \%)$ por cada una de las siguientes patologías ( $5.2 \%$ total):

a) Insuficiencia hepática por cirrosis biliar secundaria (dos meses de postoperatorio).

b) Sepsis, encefalopatía (14 días de postoperatorio).

c) Sepsis, coagulopatía (nueve días de postoperatorio).

d) Insuficiencia hepática, hipertensión portal (hepatitis C, 13 meses de postoperatorio).

A más de un año de seguimiento a partir del procedimiento inicial, 60 individuos $(89.5 \%)$ resultaron con éxito, mientras que tres $(4.5 \%)$ presentaron estenosis, por lo que requirieron una segunda intervención, sin presentar estenosis a más de un año de ella. Estos 63 en total representan 94\% con resultados satisfactorios

\section{Cuadro VII. Comorbilidades.}

Comorbilidades

Obesidad

Diabetes mellitus tipo 2

Hipertensión arterial sistémica Cirrosis biliar secundaria* Cirrosis hepático-alcohólica* Colangitis esclerosante secundaria* Hepatitis $C^{*}$

Eventración

Colestasis

Neumonía nosocomial

* De las cuatro patologías, se encontraron pacientes que fallecieron por complicaciones de las mismas. a más de un año del último procedimiento quirúrgico y definitivo.

El seguimiento fue realizado mediante revisión clínica, estudios de laboratorio y gabinete, tomando en cuenta los siguientes parámetros a estudiar:

- Clínico: ictericia, fiebre, prurito, acolia, hepatomegalia, hipertensión portal.

- Laboratorio: bilirrubinas, fosfatasa alcalina, transaminasas, panel viral de hepatitis, biopsia hepática.

- Gabinete: IDA-DISIDA, 99 Tc, resonancia magnética colangiohepatopancreática, colangiografía a través de catéter percutáneo transhepático y de sonda en T (Figuras 2 a 5). 


\section{CONCLUSIÓN}

Tanto en la literatura como en nuestra serie, los niveles más frecuentes de lesión son el tipo 3 de Bismuth/III de Stewart y Way. ${ }^{16}$

Una de las razones de éxito en la cirugía de reparación y/o reconstrucción de la vía biliar es la integridad vascular. Sin ésta, los muñones anastomóticos, así como el resto de la vía, sufren isquemia y es causa de un fracaso inminente, así como de complicaciones en el postoperatorio inmediato, con la imperativa necesidad de un tiempo quirúrgico ulterior.

Otro punto a considerar es la variabilidad anatómica de la región, presente hasta en un $15-20 \%$ de los casos, no sólo en su irrigación, sino también en la estructura a nivel de la

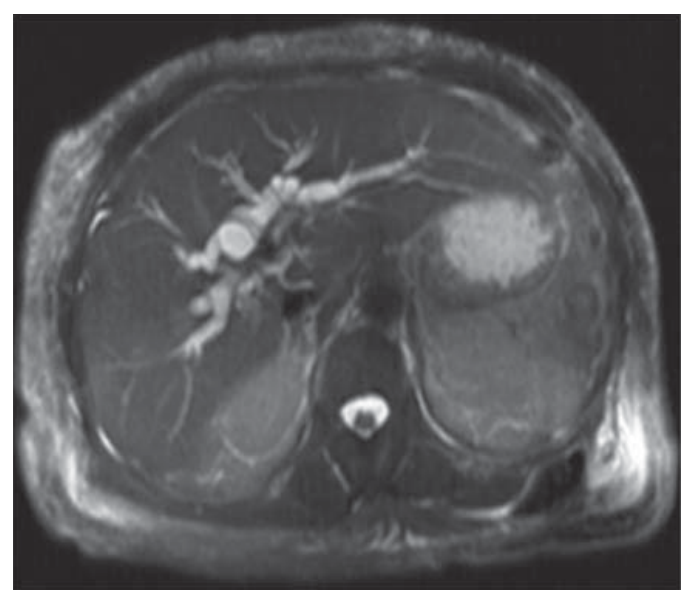

Figura 2. Resonancia magnética colangiohepatopancreática.

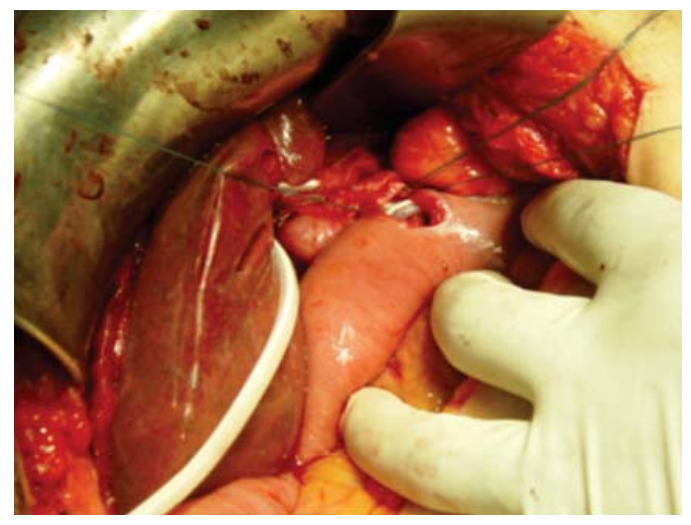

Figura 3. Hepaticoyeyunoanastomosis. confluencia de los conductos hepáticos y en la del hepático derecho. Ésta es motivo de lesión a estos niveles, y llega a poner en riesgo hasta tiempos quirúrgicos subsecuentes, como en casos de reconstrucción de la vía biliar. ${ }^{22-24}$

La muestra representada fue similar a la de Chow y colaboradores, ${ }^{49}$ quienes presentan a 69 pacientes en 15 años, con la mayor incidencia de lesiones de tipo Bismuth 1 y 3 (continuando con el tipo 2) y un manejo mediante hepaticoyeyunoanastomosis, seguida de coledocoyeyunostomía y hasta lobectomía hepática derecha.

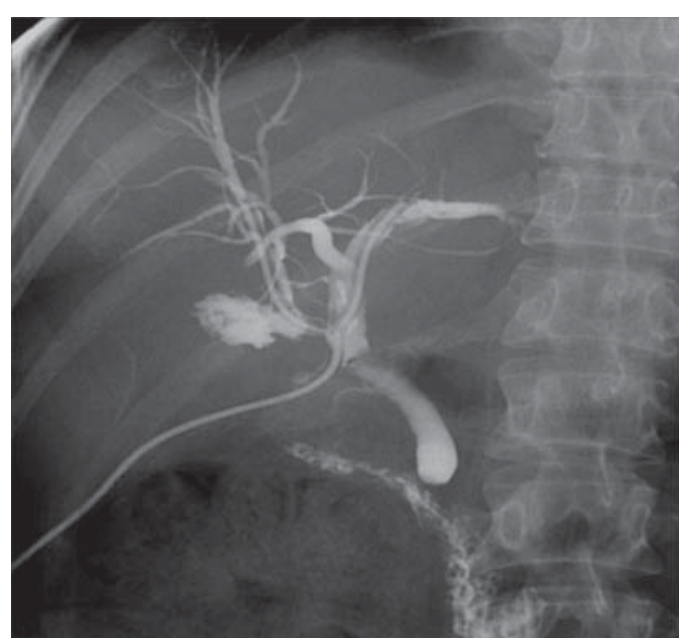

Figura 4. Colangiografía a través de catéter percutáneo transhepático.

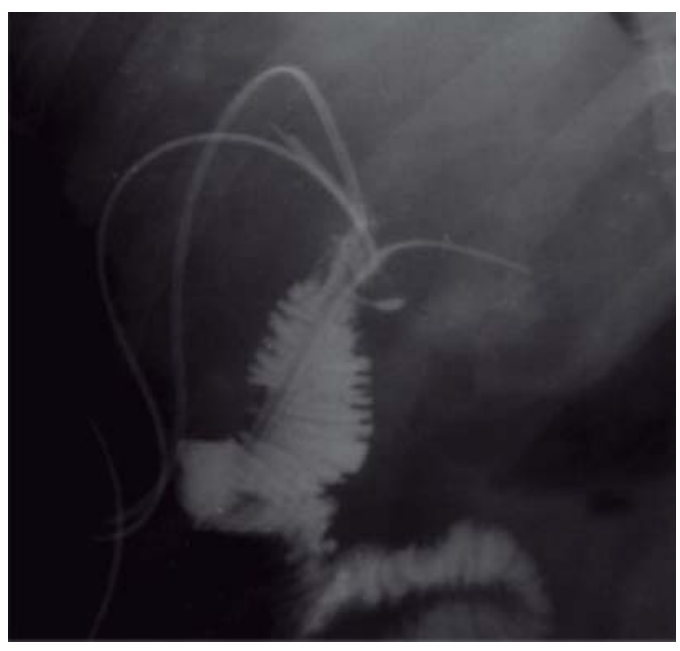

Figura 5. Colangiografía a través de sonda en T. 
Muchos autores coinciden en la experiencia en este tipo de cirugía hepatobiliar ${ }^{46-50}$ y refieren buenos resultados en aquellos pacientes enviados a unidades de tercer nivel. ${ }^{30} \mathrm{El}$ momento de referencia debe hacerse previa colocación de drenajes sin ligar conductos biliares, ${ }^{22}$ lo cual dificultaría la manipulación de los muñones de los mismos.

Compartimos un manejo similar al de Jablonska, Mercado y sus respectivos grupos, con la hepaticoyeyunoanastomosis con material absorbible 5-0, la colocación de stents transanastomóticos ${ }^{34,39}$ y la exclusión de estos últimos en aquella vía biliar no mayor a $3 \mathrm{~mm} .{ }^{37,38}$

En un manejo conjunto con otros servicios, se requiere de la experiencia de aquéllos como el de radiología intervencionista y endoscopia. ${ }^{31}$

Coincidimos con De Santibáñes y Fischer, quienes recomiendan no esperar entre un procedimiento y otro; entre seis y ocho semanas, el primero, y el segundo entre el primer procedimiento y el ulterior, ya que esto sólo predispone a la aparición de mayor incidencia de complicaciones y estancia en la unidad de cuidados intensivos. ${ }^{21,36,51,52}$ Esto se corrobora con el nivel de estenosis, cada vez más alto en las reintervenciones, lo cual compromete una elevación en la morbimortalidad, ${ }^{21,32}$ ya reflejada en tres de nuestros pacientes con una anastomosis en un nivel más alto que el anterior.

Nuestro índice de complicaciones fue de $18.7 \%$, similar a la morbilidad reportada en la literatura, del 20-30\%; la colangitis fue la más frecuente (4.3\%), seguida de infección de la herida quirúrgica, en dos pacientes (2.9\%), por debajo de lo reportado a nivel mundial (8-17.7\%).

Asimismo, la mortalidad relacionada con el evento quirúrgico se presentó en un individuo $(1.4 \%)$, concordando con otras series $(0-2 \%){ }^{31,34,40,41}$

El éxito final fue de $79.1 \%$ (53 sujetos), similar al de otras series (70-90\%), al primer año de la intervención definitiva, y de 94\% (63 personas) en total, posterior a este periodo.

Complicaciones en los primeros años, como la estenosis, sólo fueron reportadas en 4.5\% (3 enfermos), quienes a largo plazo no tuvieron el mismo desenlace. ${ }^{34,37,38,40,43,44}$

La lesión de la vía biliar es, sin duda, una de las complicaciones más peligrosas tanto para el paciente como para el cirujano. Ésta requiere del manejo en conjunto por servicios con experiencia en región hepatobiliar; su desenlace, si no llega a resolverse en un primer momento quirúrgico, conlleva una alta morbimortalidad en latencia, durante el procedimiento quirúrgico más frecuente hoy en día, la colecistectomía laparoscópica.

De vital importancia son la identificación anatómica segura, la paciencia y cautela quirúrgica, y el poder de decisión durante el acto quirúrgico para apoyarse, ya sea en el compañero quirúrgico, ya en estudios de gabinete transoperatorios y optar por la conversión a cirugía abierta con el fin de proporcionar mayor seguridad para el paciente.

\section{REFERENCIAS}

1. Bachellier P, Nakano H, Weber JC, et al. Surgical repair after bile duct and vascular injuries during laparoscopic cholecystectomy: when and how? World J Surg. 2001; 25: 1335-1345.

2. Tsalis K, Zacharakis E, Sapidis N, et al. Complications during and after laparoscopic cholecystectomy. An audit of 1042 cases. HPB (Oxford). 2005; 7 Suppl 1: 133.

3. De Reuver PR, Busch ORC, Rauws EA, et al. Longterm results of a primary end-to-end anastomosis in peroperative detected bile duct injury. J Gastrointest Surg. 2007; 11: 296-302.

4. Moossa AR, Easter DW, van Sonnenberg E, et al. Laparoscopic injuries to the bile duct. A cause for concern. Ann Surg.1992; 215: 203-208.

5. Bergman JJ, van den Brink GR, Rauws EA, et al. Treatment of bile duct lesions after laparoscopic cholecystectomy. Gut. 1996; 38: 141-147.

6. Richardson MC, Bell G, Fullarton GM. Incidence and nature of bile duct injuries following laparoscopic cholecystectomy: an audit of 5913 cases. Br J Surg. 1996; 83: 1360-1365.

7. Nuzzo G, Giuliante F, Giovannini I, et al. Bile duct injury during laparoscopic cholecystectomy: results of an Italian national survey on 56591 cholecystectomies. Arch Surg. 2005; 140: 986-992.

8. Gouma DJ, Obertop H. Management of bile duct injuries: treatment and long-term results. Dig Surg. 2002; 19: 117-122.

9. Krahenbühl L, Sclabas G, Wente MN, et al. Incidence, risk factors, and prevention of biliary tract injuries during laparoscopic cholecystectomy in Switzerland. World J Surg. 2001; 25: 1325-1330.

10. Walsh RM, Vogt DP, Ponsky JL, et al. Management of failed biliary repairs for major bile duct injuries after laparoscopic cholecystectomy. J Am Coll Surg. 2004; 199: 192-197.

11. Bruce E, Stabile MD. Laparoscopic cholecystectomy associated bile duct injuries. WJM. 1998; 168: 41.

12. Johnston GW. latrogenic bile duct stricture: an avoidable surgical hazard? Br J Surg. 1986; 73: 245-247. 
13. Smith EB. latrogenic injuries to extra hepatic ducts and associated vessels: a twenty-five year analysis. J Natl Med Assoc. 1982; 74: 735-738.

14. Keulemans YC, Bergman JJ, de Wit LT, et al. Improvement in the management of bile duct injuries? J Am Coll Surg. 1998; 187: 246-254.

15. Buell JF, Cronin DC, Funaki B, et al. Devastating and fatal complications associated with combined vascular and bile duct injuries during cholecystectomy. Arch Surg. 2002; 137: 703-710.

16. Way LW, Stewart L, Gantert W, et al. Causes and prevention of laparoscopic bile duct injuries analysis of 252 cases from a human factors and cognitive psychology perspective. Ann Surg. 2003; 237: 460-469.

17. Bektas H, Schrem H, Winny M, et al. Surgical treatment and outcome of iatrogenic bile duct lesions after cholecystectomy and the impact of different clinical classification systems. Br J Surg. 2007; 94: 1119-1127.

18. Neuhaus P, Schmidt SC, Hintze RE, et al. Classification treatment of bile duct injuries after laparoscopic cholecystectomy. Chirurg. 2000; 71: 166-173.

19. Siewert JR, Ungeheuer A, Feussner H. Bile duct lesions in laparoscopic cholecystectomy. Chirurg. 1994; 65: 748-757.

20. Alves A, Farges $\mathrm{O}$, Nicolet J, et al. Incidence and consequence of an hepatic artery injury in patients with postcholecystectomy bile duct strictures. Ann Surg. 2003; 238: 93-96.

21. De Santibáñes E, Ardiles V, Pekolj J. Complex bile duct injuries: management. HPB (Oxford). 2008; 10: 4-12.

22. Babel N, Sakpal SV, Paragi P, et al. latrogenic bile duct injury associated with anomalies of the right hepatic sectoral ducts: a misunderstood and underappreciated problem. HPB Surg. 2009; 2009: 153269.

23. Colovic RB. Isolated segmental, sectoral and right hepatic bile duct injuries. World J Gastroenterol. 2009; 15: 1415-1419.

24. Fragulidis G, Marinis A, Polydorou A, et al. Managing injuries of hepatic duct confluence variants after major hepatobiliary surgery: an algorithmic approach. World J Gastroenterol. 2008; 14: 3049-3053.

25. Ayuso JR, Ayuso C, Bombuy E, et al. Preoperative evaluation of biliary anatomy in adult live liver donors with volumetric mangafodipir trisodium enhanced magnetic resonance cholangiography. Liver Transpl. 2004; 10: 1391-1397.

26. Strasberg SM, Hertl M, Soper NJ. An analysis of the problem of biliary injury during laparoscopic cholecystectomy. J Am Coll Surg. 1995; 180: 101-125.

27. Archer SB, Brown DW, Smith CD, et al. Bile duct injury during laparoscopic cholecystectomy: results of a national survey. Ann Surg. 2001; 234: 549-559.

28. Tay KH. Laparoscopic cholecystectomy: an audit of our training programme in Singapore. HPB (Oxford). 2005; 7 Suppl 1: 136.

29. Smith EB. Complications of laparoscopic cholecystectomy. J Natl Med Assoc. 1992; 84: 880-882.

30. De Reuver PR, Grossmann I, Busch OR, et al. Referral pattern and timing of repair are risk factors for complications after reconstructive surgery for bile duct injury. Ann Surg. 2007; 245: 763-770.

31. Sicklick JK, Camp MS, Lillemoe KD, et al. Surgical management of bile duct injuries sustained during laparoscopic cholecystectomy: perioperative results in
200 patients. Ann Surg. 2005; 241: 786-792; discussion 793-795.

32. Bismuth $\mathrm{H}$, Majno PE. Biliary strictures: classification based on the principles of surgical treatment. World J Surg. 2001; 25: 1241-1244.

33. Chaudhary A, Chandra A, Negi SS, et al. Reoperative surgery for postcholecystectomy bile duct injuries. Dig Surg. 2002; 19: 22-27.

34. Jablonska B, Lampe P. latrogenic bile duct injuries: etiology, diagnosis and management. World J Gastroenterol. 2009; 15: 4097-4104.

35. Tocchi A, Mazzoni G, Liotta G, et al. Late development of bile duct cancer in patients who had biliary-enteric drainage for benign disease: a follow-up study of more than 1,000 patients. Ann Surg. 2001; 234: 210-214.

36. Fischer $\mathrm{CP}$, Fahy BN, Aloia TA, et al. Timing of referral impacts surgical outcomes in patients undergoing repair of bile duct injuries. HPB (Oxford). 2009; 11: 32-37.

37. Lillemoe KD, Melton GB, Cameron JL, et al. Postoperative bile duct strictures: management and outcomes in the 1990s. Ann Surg. 2000; 232: 430-441.

38. Lillemoe KD, Martin SA, Cameron JL, et al. Major bile duct injuries during laparoscopic cholecystectomy. Follow-up after combined surgical and radiologic management. Ann Surg. 1997; 225: 459-471.

39. Mercado MA, Chan $\mathrm{C}$, Orozco H, et al. To stent or not to stent bilioenteric anastomosis after iatrogenic injury: a dilemma not answered? Arch Surg. 2002; 137: 60-63.

40. Hall JG, Pappas TN. Current management of biliary strictures. J Gastrointest Surg. 2004; 8: 1098-1110.

41. Ahrendt SA, Pitt HA. Surgical therapy of iatrogenic lesions of biliary tract. World J Surg. 2001; 25: 13601365.

42. Johnson SR, Koehler A, Pennington LK, et al. Long-term results of surgical repair of bile duct injuries following laparoscopic cholecystectomy. Surgery. 2000; 128: 668-677.

43. Tocchi A, Costa G, Lepre L, et al. The long-term outcome of hepaticojejunostomy in the treatment of benign bile duct strictures. Ann Surg. 1996; 224: 162-167.

44. Terblanche J, Worthley CS, Spence RA, et al. High or low hepaticojejunostomy for bile duct strictures? Surgery. 1990; 108: 828-834.

45. Boerma D, Rauws EA, Keulemans YC, et al. Impaired quality of life 5 years after bile duct injury during laparoscopic cholecystectomy: a prospective analysis. Ann Surg. 2001; 234: 750-757.

46. De Reuver PR, Sprangers MA, Gouma DJ. Quality of life in bile duct injury patients. Ann Surg. 2007; 246 :161-163.

47. Andersson R, Eriksson K, Blind PJ, et al. latrogenic bile duct injury - a cost analysis. HPB (Oxford). 2008; 10: 416-419.

48. Savader SJ, Lillemoe KD, Prescott CA, et al. Laparoscopic cholecystectomy-related bile duct injuries: a health and financial disaster. Ann Surg. 1997; 225: 268-273.

49. Chow SC, Sahajpal AK, Dixon E, et al. Bile duct injuries associated with laparoscopic cholecystectomy: timing of repair determines long-term outcomes. Can J Surg. 2008; 51 Suppl: 42. 
50. Silva MA, Coldham C, Mayer AD, et al. Specialist outreach service for on-table repair of iatrogenic bile duct injuries - a new kind of "travelling surgeon". Ann R Coll Surg Engl. 2008; 90: 243-246.

51. Pekolj J. Manejo de las complicaciones más frecuentes de la cirugía abdominal. Rev Arg Cirg. 2003 [Número extraordinario].
52. De Santibañés E, Sivori J, Pekolj J, et al. Lesiones de la vía biliar secundaria a colecistectomía laparoscópica. Rev Arg Cirg. 1996; 70: 208-219.

Correspondencia:

Jorge Elorza Bonifaz Calvo

E-mail: george_jebc@hotmail.com 